\title{
Design of Two-Stage Comb-Based Decimation Filter with High Aliasing Rejection and Low Passband Droop
}

\author{
Gordana Jovanovic Dolecek \\ Department of Electronics, Institute INAOE, Puebla, Mexico \\ Email: gordana@ieee.org
}

How to cite this paper: Dolecek, G.J. (2021) Design of Two-Stage Comb-Based Decimation Filter with High Aliasing Rejection and Low Passband Droop. Journal of Computer and Communications, 9, 169-179. https://doi.org/10.4236/jcc.2021.99014

Received: September 9, 2021

Accepted: September 27, 2021

Published: September 30, 2021

\begin{abstract}
This paper presents a simple method for the design of comb-based decimation filter with a high aliasing rejection and low passband droop. The method is based on the application of the mathematical result on zeros of symmetric polynomials on the unit circle. The application of this result leads to the introduction of the four additional single zeros into comb folding bands, where aliasing occur. As a result, the folding bands become wider and the aliasing rejection is increased. The passband droop is compensated by a simple compensator who works at low rate. The comparisons with the most recent methods from literature confirmed the advantages of this proposal regarding magnitude responses and the required complexity.
\end{abstract}

\section{Keywords}

Decimation, Comb Filter, Aliasing, Compensator, Passband Droop

\section{Introduction}

Decimation is the process of decreasing the sampling rate in the digital domain by an integer, called downsampling factor. This process is composed of two stages. In the first stage is the decimation filter while in the second stage the sampling rate is decreased. The decimation filter is needed to prevent aliasing which occurs during the decreasing the sampling rate. Decimation has many applications in communications, software defined radio, Sigma-Delta Analog-Digital Converters, among others [1].

The most simple decimation filter is a comb filter, which has all its coefficients equal to unity. The system function of comb filter is given as: 


$$
H(z)=\frac{1-z^{-M}}{M\left(1-z^{-1}\right)},
$$

where $M$ is the decimation factor.

Comb filter naturally provides the attenuation in the frequency bands around comb zeros, called folding bands. However, this attenuation is not enough in many applications. This attenuation could be increased by cascading $K$ combs, where $K$ is called the comb order. However, the increase of $K$ increases the droop in the passband, which may result in the deterioration of the decimated signal.

Different methods have been proposed to increase the aliasing rejection in comb filters [2]-[11]. Presti in [2] proposed Rotated Sinc (RS) filter obtained by the rotation of the comb zeros around their original positions in the folding bands. As a result, the folding bands become wider, thus providing increased attenuation in the folding bands. The drawback of this approach is the introduction of two multipliers, and possible instability when the coefficients of RS filter are presented with finite precision. The authors in [3] [4] [5] used simple multiplierless filters to get the similar effect as in RS filters but without the mentioned drawbacks. Laddomada in [3] used the cyclotomic polynomials, while the authors in [4] [5] explored symmetric polynomials to separate comb zeros [4], or to introduce the additional zeros into comb folding bands [5]. Similarly, the sharpening techniques were proposed in [6]-[11]. Willson at all introduced in [6] the simple sharpening polynomial to improve the passband and the stopband comb magnitude characteristic. The authors in [7] proposed a two-stage structure where the sharpening is performed only at the second stage. Coleman in [8] proposed sharpening using Chebyshev polynomials. In [9] [10] are proposed sharpened polynomials to increase comb aliasing rejection and the novel compensators to compensate high passband droop in sharpened comb filters. In [11] was proposed a linear programming optimization to find the sharpening coefficients and improve comb magnitude response in a two stage structure.

The goal of this work is to propose an alternative method to improve comb magnitude characteristic. The proposed method is based on the modification of work in [5] and the simple compensator working at low rate.

The rest of the paper is organized as follows. Next Section introduces the proposed filter. The design of compensators is elaborated in Section 3. Finally, the comparisons with some recent methods from literature are given in Section 4.

\section{Proposed Filter}

In [5] was proposed the following decimation filter,

$$
H_{m}(z)=H^{K-2}(z) G(z),
$$

where $K$ is the order of the equivalent comb filter, $H(z)$ is the comb filter given in (1) and $G(z)$ is given as:

$$
G(z)=\left[1+\lambda_{1} \sum_{k=1}^{M-2} z^{-k}+z^{-(M-1)}\right] \times\left[1+\lambda_{2} \sum_{k=1}^{M-2} z^{-k}+z^{-(M-1)}\right],
$$


where $\lambda_{1}=2^{-k}$ and $\lambda_{2}=1+\lambda_{1}$, and $k$ is an integer.

The filter (3) introduces two additional zeros into comb folding bands. However, for high values of $M$ and $K$, the effect of the introduced zeros is low.

We propose here a two-stage structure, in which the modified filter (3) is applied only in the second stage as described in the following section.

\subsection{Increasing Aliasing Rejection}

It is supposed that the decimation factor $M$ can be presented as a product of two integers, $M=M_{1} M_{2}$. The system function of the comb (1), can be presented as:

$$
H(z)=H_{1}(z) H_{2}\left(z^{M_{1}}\right)
$$

where:

$$
H_{1}(z)=\frac{1-z^{-M_{1}}}{M_{1}\left(1-z^{-1}\right)} ; H_{2}\left(z^{M_{1}}\right)=\frac{1-z^{-M}}{M_{2}\left(1-z^{-M_{1}}\right)}
$$

We consider a two-stage comb structure in which a comb filter $H_{1}(z)$ is decimated by $M_{1}$ in the first stage, while in the second stage is a comb filter $H_{2}(z)$ decimated by $M_{2}$.

We propose to introduce a modified comb filter, derived from (3) and denoted as $H_{m}(z)$, only at the second stage:

$$
H_{m}(z)=\left[1+2^{-1} \sum_{i=1}^{M_{2}-2} z^{-i}+z^{-\left(M_{2}-1\right)}\right]^{2} \times\left[1+\left(1+2^{-1}\right) \sum_{i=1}^{M_{2}-2} z^{-i}+z^{-\left(M_{2}-1\right)}\right]^{2} .
$$

Using (5) and (6), the system function of the proposed filter $H_{p}(z)$ is given as:

$$
H_{p}(z)=H_{1}^{K_{1}}(z) H_{2}^{K_{2}}\left(z^{M_{1}}\right) H_{m}\left(z^{M_{1}}\right),
$$

where $K_{1}$ and $K_{2}$ are orders of the combs $H_{1}(z)$ and $H_{2}(z)$, respectively. The values of $K_{1}$ and $K_{2}$ are chosen to give high aliasing attenuation, and are given in Table 1. The method is illustrated in the following example.

Example 1: We consider $M=12, K_{1}=6, K_{2}=1$, and three different values for $M_{2}: 3,4$ and 6 . The magnitude responses of the proposed filter and the equivalent comb of the order $K=5$, are shown in Figure 1.

\subsection{Choice of $M_{1}$ and $M_{2}$}

Observing Figure 1 we can see that the choice of $M_{2}$ affects the magnitude response.

Table 1. Values of $K_{1}$ and $K_{2}$.

\begin{tabular}{cc}
\hline$K_{1}$ & $K_{2}$ \\
\hline 5 & 1 \\
6 & 1,2 \\
7 & 2 \\
\hline
\end{tabular}




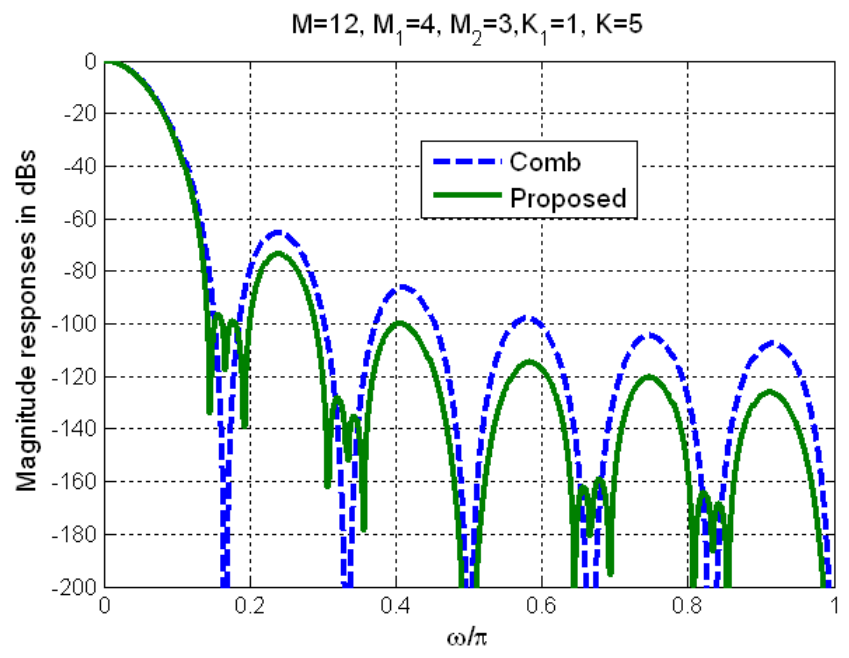

(a)

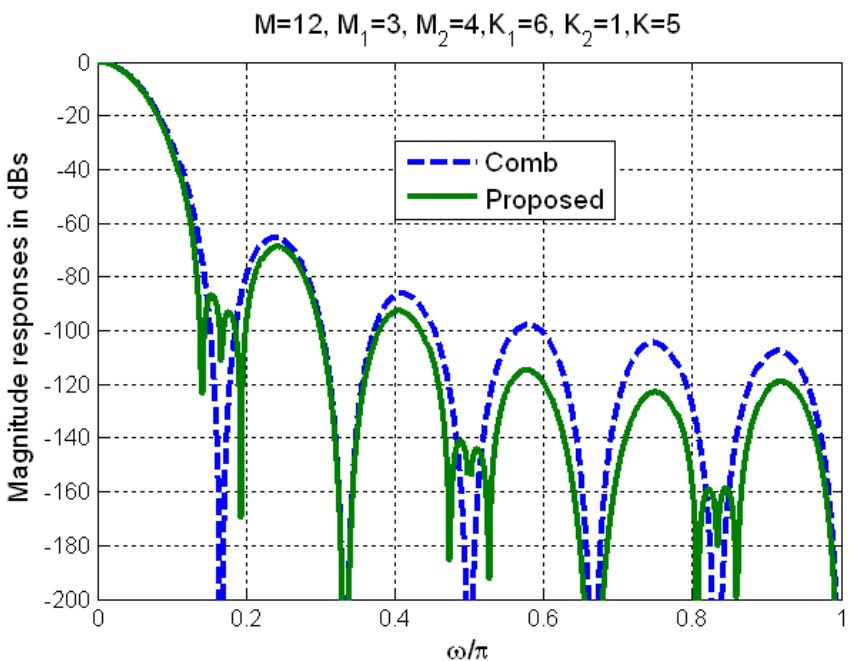

(b)

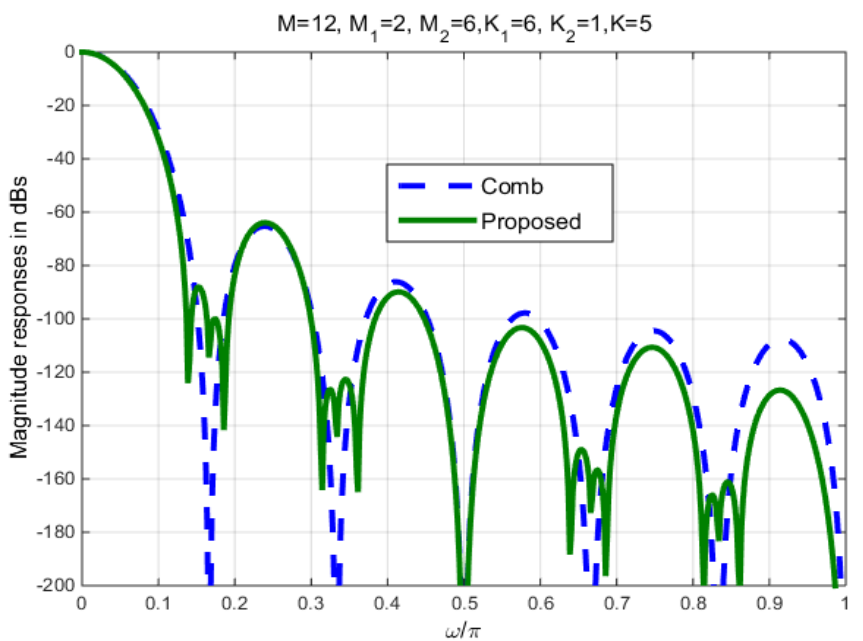

(c)

Figure 1. Magnitude responses in Example 1. (a) $M_{1}=4, M_{2}=3$; (b) $M_{1}=3, M_{2}=4$; (c) $M_{1}=2, M_{2}=6$. 
If $M_{2}$ is odd, then each $M_{2}$-th folding band is not improved. However, if $M_{2}$ is even, then each $M_{2} / 2$-th folding band is not improved.

Therefore, the value $M_{2}$ should be chosen in order that more folding bands are improved. As a result, in Example 1 the choice $M_{2}=3$ and $M_{2}=6$ (every third band is not improved) are better than the choice $M_{2}=4$ (every second band is not improved).

From the other side, the value of $M_{1}$ affects the complexity. Higher values of $M_{1}$ result that the second stage works at lower rate.

Therefore the choice of $M_{1}$ and $M_{2}$ must be a compromise between the high aliasing rejection and low complexity.

In Example 1 the best choice is $M_{1}=4$ and $M_{2}=3$.

\subsection{Complexity}

The complexity is expressed in number of APOS (adders per output sample), [12].

The number of $A P O S$ for the proposed filter is presented in the following equation:

$$
\text { APOS }=K_{1} M+\left(K_{1}-K_{2}\right) M_{2}+M_{2}\left(2 M_{2}-1\right)+K_{2} .
$$

Using (8) we find the following values of APOS for given values of $M_{1}$ and $M_{2}$ in the Example 1.

$$
\begin{aligned}
& M_{1}=4, M_{2}=3: \text { APOS }=103 . \\
& M_{1}=3, M_{2}=4: \text { APOS }=121 . \\
& M_{1}=2, M_{2}=6: A P O S=169 .
\end{aligned}
$$

Note that the increase of $M_{1}$ results in the decrease of number of APOS.

\section{Compensation}

Since the passbands in the proposed filter and the equivalent comb are similar, we propose to adapt the compensator from [13]. The compensator in [13] provides a good compensation of the comb passband droop requiring a low number of adders. The magnitude response of the compensator is given as:

$$
C\left(e^{j \omega M}\right)=\left[1+A \sin ^{4}(\omega M / 2)\right] \times\left[1+B \sin ^{2}(\omega M / 2)\right],
$$

where $A$ and $B$ are the amplitudes of sinusoidal functions.

The total number of adders of compensator $N_{a}$ is given as [13]:

$$
N_{a}=9+N_{A}+N_{B},
$$

where $N_{A}$ and $N_{B}$ are the total number of adders for the parameters $A$ and $B$, respectively, which are presented in a SPT form. The compensator works at low rate, i.e. after the decimation by $M$. The system function of the proposed compensated filter is given as:

$$
H_{p c}(z)=H_{p}(z) C\left(z^{M}\right)=H_{1}^{K_{1}}(z) H_{2}^{K_{2}}\left(z^{M_{1}}\right) H_{m}\left(z^{M_{1}}\right) C\left(z^{M}\right),
$$

where $H_{p}(z)$ is given in (7) and $C(z)$ is the system function of the compensator.

Using (8), the total number of $A P O S_{C}$ in the proposed compensated filter is 
equal to:

$$
A P O S_{C}=A P O S+N_{A}=K_{1} M+\left(K_{1}-K_{2}\right) M_{2}+M_{2}\left(2 M_{2}-1\right)+K_{2}+N_{a} .
$$

where $N_{a}$ is given in (10).

As a difference to [13], the values $A$ and $B$ are obtained here using the Particle Swarm Optimization (PSO) of MATLAB. Particularly, the MATLAB function particleswarm. $m$ is used. The procedure of using particleswarm.m to get the parameters $A$ and $B$ for a given $M$, and $K$ is presented in [14], and is not repeated here. The only difference is that here the optimization is performed for the proposed filter (11) and in [14] for the comb filter.

As an example, Table 2 presents the values of $A$ and $B$ in the SPT form for $M$ $=12$ and the values $K_{1}$ and $K_{2}$ from Table 1 . The total number of adders $N_{a}$ and the absolute value of the maximum passband deviation $\delta$ in dBs, are also shown.

Next example compares the magnitude responses of the proposed filter from Example 1, and the proposed compensated filter.

Example 2. In this example all parameters are equal as in Example 1. The parameters of compensator are obtained from the second row in Table 2 and are equal: $A=2^{0}-2^{-3}-2^{-7}, B=2^{0}-2^{-2}+2^{-4}$, requiring 13 adders. From (12) the number of $A P O S_{C}$ is equal to 116. The magnitude responses are shown in Figure 2.

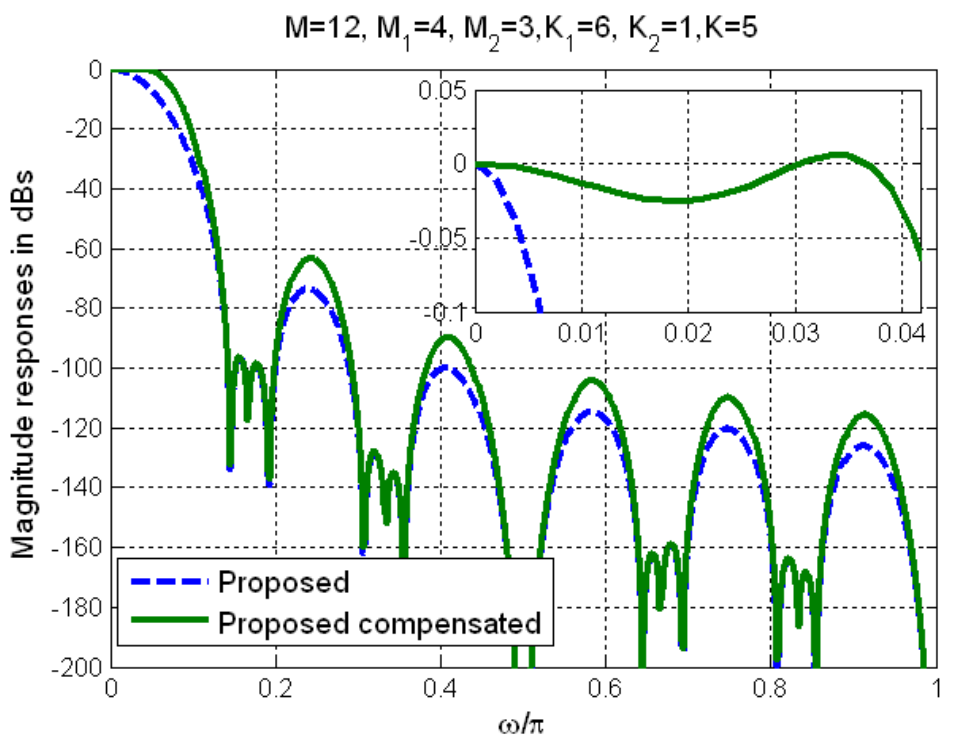

Figure 2. Magnitude responses of the compensated and the original proposed filter.

Table 2. Values of $A, B, N_{a}$ and $\delta$.

\begin{tabular}{cccccc}
\hline$K_{1}$ & $K_{2}$ & $A$ & $B$ & $N_{a}$ & $\delta$ \\
\hline 5 & 1 & $2^{0}-2^{-5}$ & $2^{0}-2^{-2}$ & 11 & 0.054 \\
6 & 1 & $2^{0}-2^{-3}-2^{-7}$ & $2^{0}-2^{-2}+2^{-4}$ & 13 & 0.05 \\
6 & 2 & $2^{0}+2^{-7}$ & $2^{0}$ & 10 & 0.062 \\
7 & 2 & $2^{0}+2^{-6}$ & $2^{0}+2^{-5}$ & 11 & 0.068 \\
\hline
\end{tabular}




\section{Comparisons}

In this section we compare the proposed filter with the methods from literature regarding magnitude response and complexity expressed in APOS.

\subsection{Comparison with Method in [5]}

We pick up $M=12$ and $K=4$ in the method in [5]. In the proposed method $M_{1}$ = 4, $M_{2}=3, K_{1}=5$ and $K_{2}=1$. The parameters of compensator are used from the first row in Table 2.

The overall magnitude responses and the passband zooms are contrasted in Figure 3. Observe that the proposed compensated filter provides better aliasing rejection and an improved passband.

Additionally, the proposed method has low complexity, requiring the number of $A P O S_{C}=99$, while the method in [5] requires 302 numbers of $A P O S$.

\subsection{Comparison with Method in [4]}

For a sake of comparison we chose $M=15$ and $K=5$ in the method proposed in [4]. In the proposed method $M_{1}=5, M_{2}=3, K_{1}=6$, and $K_{2}=2$. The parameters of compensator are $A=2^{0}+2^{-5}$ and $B=2^{0}$.

The number of APOS in method [4] is equal to 123, while in the proposed method is equal to 119 (without compensator), and 129 (with compensator).

The magnitude responses are compared in Figure 4. Note that the proposed method provides much better magnitude characteristic.

\subsection{Comparison with Method in [9]}

The authors in [9] proposed various sharpened polynomials to improve the

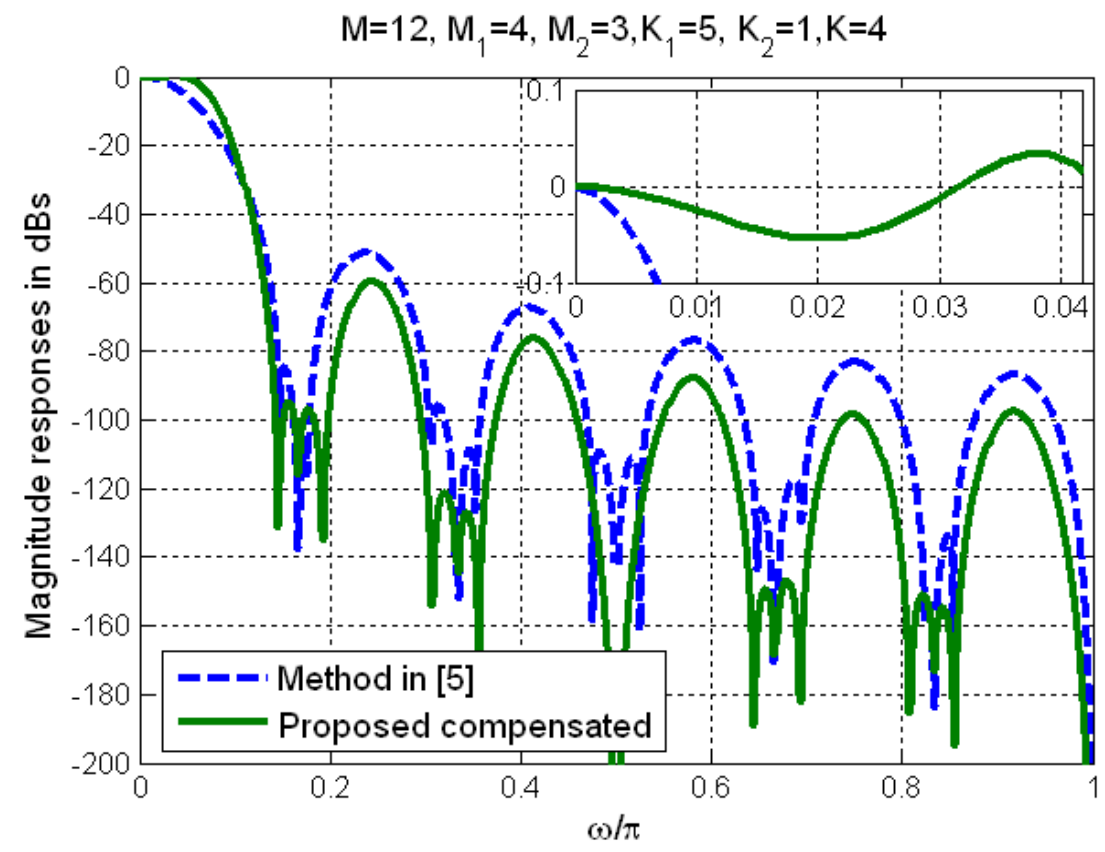

Figure 3. Comparison with method in [5]. 


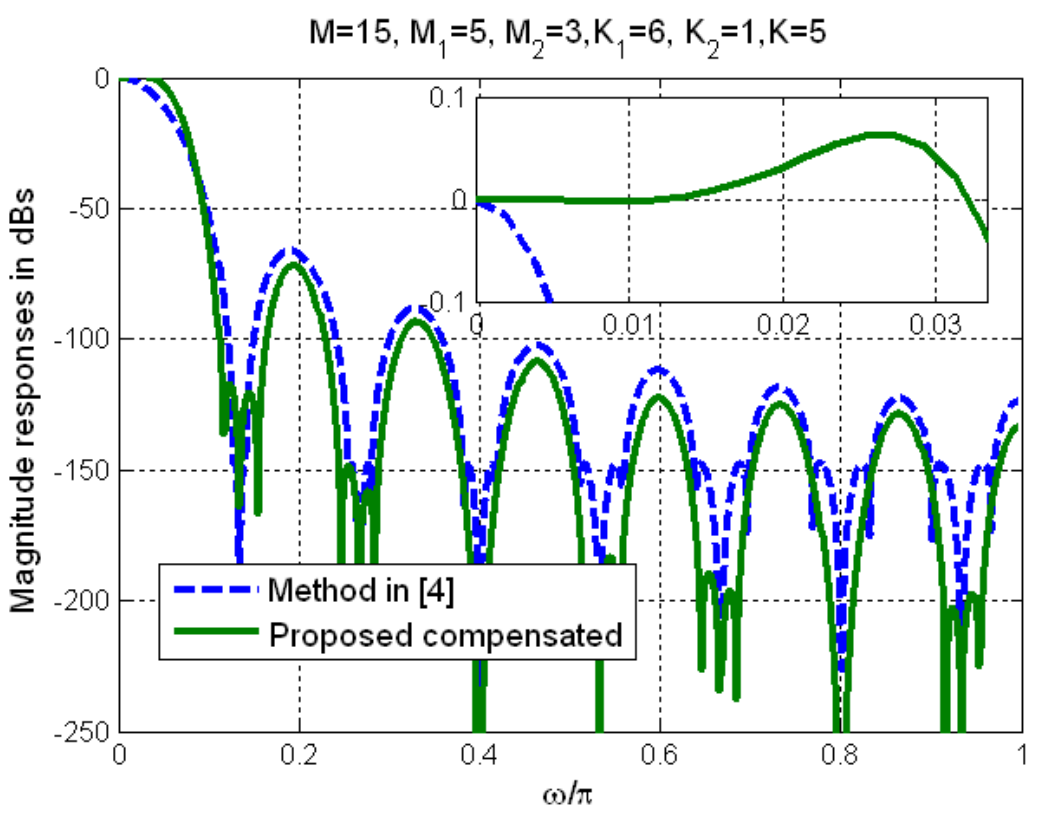

Figure 4. Comparison with method in [4].

comb aliasing rejection. Since sharpened combs exhibit a high passband droop, the designs of the compensators were also proposed in [9].

In this comparison we chose $M=32$ and the sharpening polynomial in [9] $p(x)=-2^{-7} x^{2}+x^{4}$, where $x$ is the comb filter. The coefficients of compensator are given in Table in [9].

In the proposed filter $M_{1}=8, M_{2}=4, K_{1}=5$, and $K_{2}=1$. The parameters of compensator are $A=2^{0}-2^{-8}$, and $B=2^{0}-2^{-2}$, requiring $N_{a}=11$ adders.

The number of APOS in the filter proposed in [9] is equal to 233, while in the proposed method is equal to 216 .

The magnitude responses are shown in Figure 5. We can observe that the proposed filter provides better aliasing rejection and better passband characteristic, requiring lesser number of APOS.

\subsection{Comparison with Method in [11]}

Authors in [11] proposed a two-stage decimation filter. In the first stage is a comb filter of order $Q$, while in the second stage is a sharpened comb. The coefficients of the sharpened polynomial are obtained using LP optimization and are given in Table in [11].The parameters of design in this comparison in method [11] are: $M=32$ and $M_{1}=4, M_{2}=8, Q=8$. In the proposed method $M_{1}=8$, while $M_{2}=4, K_{1}=7, K_{2}=2$. The parameters of compensator are $A=2^{0}+2^{-4}$, and $B=2^{0}+2^{4}$, requiring 11 adders.

The magnitude responses are compared in Figure 6.

The number of APOS in [11] is equal 403, while in the proposed method is equal to 285 .

We can see that the proposed method provides better aliasing rejection, as well as better passband characteristic, requiring lesser number of APOS. 


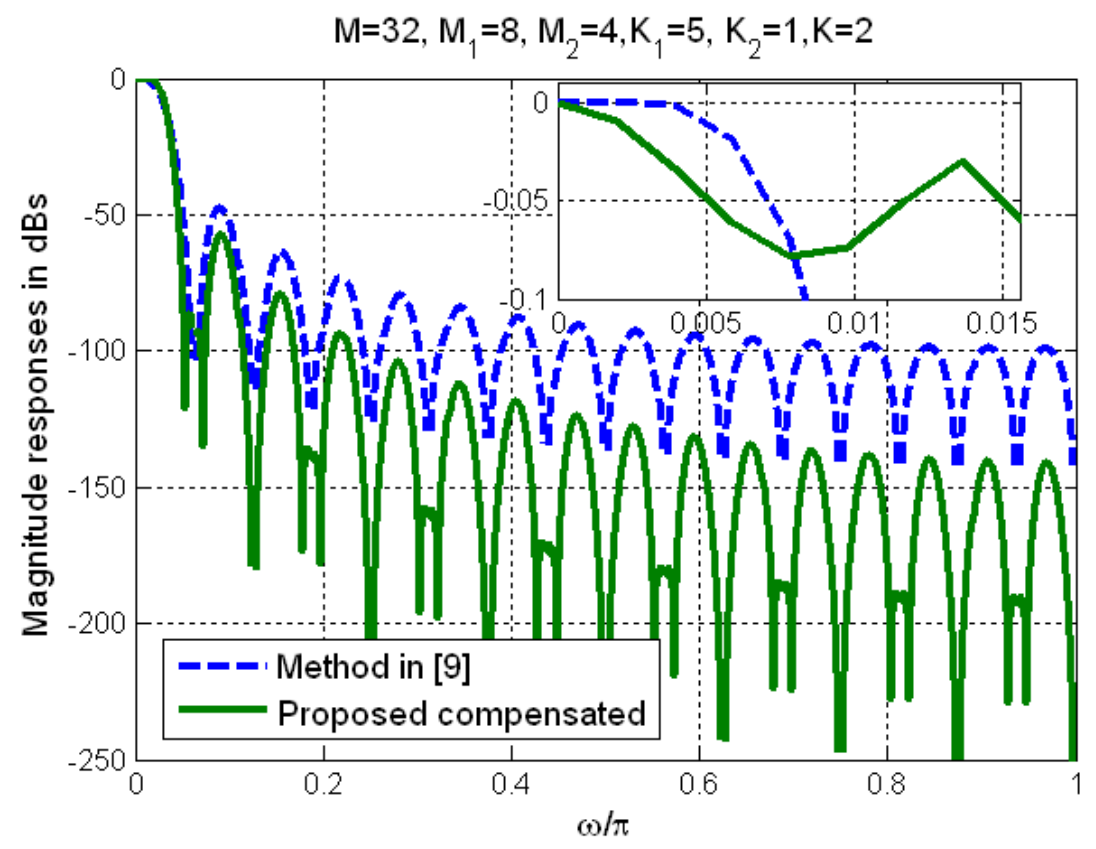

Figure 5. Comparison with method in [9].

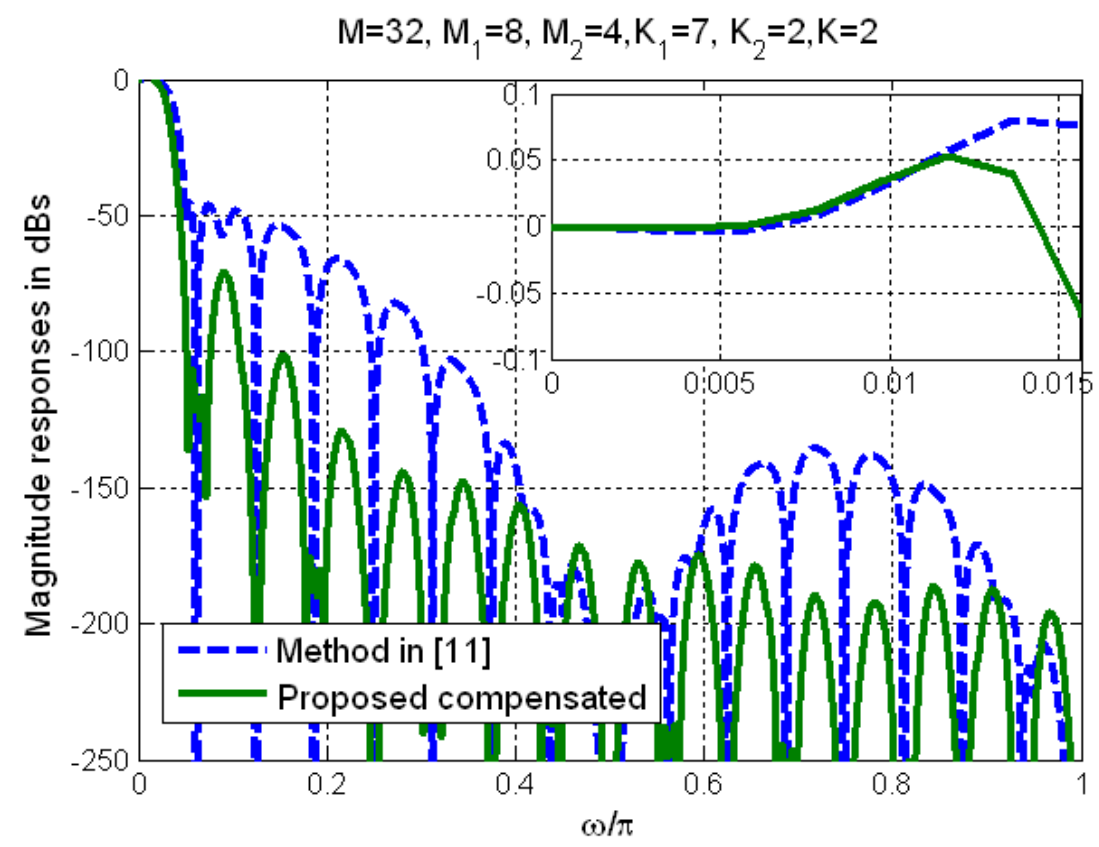

Figure 6. Comparison with method in [11].

\section{Conclusions}

We present a simple design of a two stage decimation filter with high aliasing rejection and good passband compensation.

The aliasing rejection is increased by introducing the modified comb at the second stage. As a result, four additional zeros are inserted into certain comb folding bands, thus providing increases of the folding bands widths, and increases of aliasing rejection in folding bands. The attenuation in folding bands is 
higher than $80 \mathrm{dBs}$.

The decimation factor at the second stage determines which folding band gets additional zeros. On the other side, the complexity of the filter, expressed in $A P O S$ is determined by the decimation factor at the first stage.

Usually, there is a compromise in choosing the decimation factors in the first and second stages.

The passband is improved by the simple multiplierless compensator working at low rate. The parameters of compensator are obtained by PSO. The absolute value of the maximum passband deviation is lesser than $0.08 \mathrm{dBs}$, and depends on the values of decimation factors and the orders of comb filters, at the first and second stages.

The comparisons with methods from literature demonstrated that the proposed filter provides better magnitude characteristic, while requires lesser number of $A P O S$.

\section{Conflicts of Interest}

The author declares no conflicts of interest regarding the publication of this paper.

\section{References}

[1] Jovanovic Dolecek, G. (2003) Introduction to Multirate Systems. In: Jovanovic Dolecek, G., Ed., Multirate Systems. Design and Application, IGP, Hershey. https://doi.org/10.4018/978-1-93070-830-3

[2] Presti, L.L. (2000) Efficient Modified-Sinc Filters for Sigma-Delta A/D Converters. IEEE Trans. on Circ. and Syst. II: Analog and Dig. Sig. Proc., 47, 1204-1213. https://doi.org/10.1109/82.885128

[3] Laddomada, M. (2008) Design of Multistage Decimation Filters Using Cyclotomic Polynomials: Optimization and Design Issues. IEEE Trans. on Circ. and Syst I: Reg. papers, 55, 1977-1987. https://doi.org/10.1109/TCSI.2008.918193

[4] Jovanovic Dolecek, G. and Dolecek, L. (2016) Exploiting Features of Symmetric Polynomials for Improved Comb Filter Design. Proc. IEEE Int. Conf. Sig. Proc.: Alg., Arch, Arrang., and Appl (SPA 2016), Poznan, 26-29. https://doi.org/10.1109/SPA.2016.7763581

[5] Jovanovic Dolecek, G. (2017) Improving Comb Alias Rejection by Exploiting Certain Class of Palindromic Polynomials. Proc of 23 Europ Conf on Circ Th and Design (ECCTD 2017), Catania, Sept. 2017, 1-4. https://doi.org/10.1109/ECCTD.2017.8093347

[6] Kwentus, A., Jiang, Z. and Wilson, A.N. (1997) Application of Filter Sharpening to Cascaded-Integrator-Comb Decimation Filters. IEEE Trans. Sig. Proc, 45, 457-467. https://doi.org/10.1109/78.554309

[7] Jovanovic Dolecek, G. and Mitra, S.K. (2005) A New Two-Stage Sharpened Comb Decimator. IEEE Trans. on Circ. and Syst I: Reg. Papers, 52, 1414-1420. https://doi.org/10.1109/TCSI.2005.851390

[8] Coleman, O.J. (2012) Chebyshev Stopbands for CIC Decimation Filters and CIC Implemented Array Tappers in 1D and 2D. IEEE Trans. on Circ. and Syst I: Reg. papers, 59, 2956-2968. https://doi.org/10.1109/TCSI.2012.2206435 
[9] Molnar, G., Dudarin, A. and Vucic, M. (2018) Design and Multiplierless Realization of Maximally Flat Sharpened CIC Compensator. IEEE Trans on Circ and Syst-II: Express Briefs, 1, 51-55. https://doi.org/10.1109/TCSII.2017.2700081

[10] Dudarin, A., Molnar, G. and Vucic, M. (2018) Optimum Multiplierless Compensators for Sharpened Cascaded Integrator-Comb Decimation Filters. El Lett, 16, 971-972. https://doi.org/10.1049/el.2018.5114

[11] Gautam, D., Khare, K. and Shrivastava, B.P. (2021) A Novel Approach for Optimal Design of Sample Rate Conversion Filter Using Linear Optimization Technique, IEEE Access, 9, 44436-44441. https://doi.org/10.1109/ACCESS.2021.3066292

[12] Abu-Al-Saud, W.A. and Stuber, G.L. (2006) Efficient Sample Rate Conversion in Software Radio Systems. IEEE Trans. Sig. Proc, 54, 932-939.

https://doi.org/10.1109/TSP.2005.861737

[13] Jovanovic Dolecek, G., Garcia Baez, R., Molina Salgado, G. and de la Rosa, J.M. (2017) Novel Multiplierless Wideband Comb Compensator with High Compensation Capability. Circ. Syst. and Sig. Proc., 36, 2031-2049.

https://doi.org/10.1007/s00034-016-0398-0

[14] Salguero Luna, S.A. and Jovanovic Dolecek, G. (2021) Increasing Flexibility of the Comb Compensator Design Using Particle Swarm Optimization. Ingeria, Invest. $y$ Techn., XII, 1-12. (In Spanish) https://doi.org/10.22201/fi.25940732e.2021.22.2.014 